\title{
Métodos de correção da produtividade de grãos de milho relacionados à adaptabilidade e estabilidade de cultivares
}

\author{
Alberto Cargnelutti Filho(1) ${ }^{(1)}$ Lindolfo Storck ${ }^{(2)}$ \\ (1)Fundação Estadual de Pesquisa Agropecuária, Rua Gonçalves Dias, 570, Bairro Menino Deus, CEP $90130-060$ Porto Alegre, RS. E-mail: \\ alberto-cargnelutti@fepagro.rs.gov.br (2)Universidade Federal de Santa Maria, Dep. de Fitotecnia, Avenida Roraima, s/n, Bairro Camobi, CEP \\ 97105-900 Santa Maria, RS. E-mail: storck@ccr.ufsm.br
}

\begin{abstract}
Resumo - O objetivo deste trabalho foi verificar a interferência de métodos de correção da produtividade de grãos de milho nos parâmetros adaptabilidade e estabilidade de cultivares. A correção da produtividade de grãos foi avaliada em 31 ensaios de competição de cultivares de milho, por meio dos seguintes métodos: sem correção, regra de três, método de Zuber, covariância da população média, covariância da população ideal, método proposto por Cruz, método proposto por Vencovsky \& Cruz e método de correção estratificada. As estatísticas, desvio quadrático médio para cada um dos parâmetros de adaptabilidade e estabilidade e índice de concordância, foram utilizadas para verificar a magnitude da influência das correções. Os métodos covariância para a população média, covariância para a população ideal, Vencovsky \& Cruz e correção estratificada são os que acarretam menores discordâncias dos parâmetros de adaptabilidade e estabilidade de cultivares em relação aos dados sem correção.
\end{abstract}

Termos para indexação: Zea mays, covariância, uniformidade, análise estatística, precisão experimental.

\section{Grain yield correction methods related to adaptability and stability of corn cultivars}

\begin{abstract}
The objective of this research was to verify if grain yield corrections could interfere in adaptability and stability parameters of corn cultivars. Yield corrections of 31 corn competition trials were done, based on plant populations. Actual data was compared with corrected data based on plant average yield, Zuber method, mean population covariance, recommended population covariance, Cruz method, Vencovsky \& Cruz method and stratified correction method. The magnitude of correction interferences was verified using the statistics of square mean deviations of each adaptability and stability parameters and concordance index. Corrections based upon mean and recommended population covariance, Vencovsky \& Cruz methods and stratified method have the lowest discordance compared to adaptability and stability parameters of corn cultivars of actual grain yield data.
\end{abstract}

Index terms: Zea mays, covariance, uniformity, statistics analysis, experimental precision.

\section{Introdução}

A variação entre unidades experimentais constitui uma estimativa do erro experimental (Ramalho et al., 2000; Storck et al., 2000a), com interferência direta nos testes de hipótese e nos procedimentos que dizem respeito às comparações múltiplas de médias. Em ensaios de competição de cultivares, deve-se manter as condições experimentais o mais uniforme possível. Para isso, necessita-se de cuidados na implantação, na condução dos experimentos e na coleta dos dados, visando garantir que a superioridade de uma cultivar em relação a outra reflita sua superioridade genética (Cruz \& Carneiro, 2003).
O uso dos princípios básicos da experimentação no planejamento, a condução e a análise adequadas de experimentos são fundamentais para se manter o erro experimental em níveis aceitáveis (Steel et al., 1997; Storck et al., 2000a; Cruz \& Carneiro, 2003). Outras formas de minimizar o efeito do erro experimental e de discriminar melhor as diferenças entre os tratamentos avaliados são o uso de maior número de repetições, e parcelas menores (Gomes, 1994; Conagin et al., 1995; Storck et al., 2000a), o uso de unidades experimentais homogêneas e a realização, de maneira uniforme, dos tratos culturais inerentes à cultura no decorrer do experimento (Lopes \& Storck, 1995; Lúcio, 1997). Além disso, 
Cruz \& Carneiro (2003) incluem a mensuração de variáveis auxiliares, ou covariáveis, relacionadas às variáveis principais, como uma das técnicas experimentais utilizadas para reduzir a variação ambiental. Assim, a correção prévia dos dados quanto à desuniformidade na população de plantas consiste em uma forma de se fazer comparações mais apropriadas sobre a superioridade genotípica (Cruz \& Carneiro, 2003). A magnitude do erro experimental e o atendimento às pressuposições do modelo matemático (Storck et al., 2000a) servem para avaliar a qualidade de um experimento. E ainda, as estatísticas, coeficiente de variação (Scapim et al., 1995) e diferença mínima significativa, calculadas pelo método de Tukey (Lúcio, 1997), são consideradas adequadas para classificar a precisão experimental.

A precisão experimental dos ensaios de competição de cultivares de milho é baixa (Lopes, 1993; Lopes \& Storck, 1995; Lúcio, 1997; Marques, 1999), ocasionando a discriminação ineficiente entre as cultivares, o que pode induzir a conclusões incorretas em relação às cultivares a serem indicadas aos produtores. A análise da covariância com a produtividade de grãos, usando-se como covariável o número de plantas na colheita (Veronesi et al., 1995; Storck et al., 2000b; Schmildt et al., 2001), os resultados de análise de solo (Feijó et al., 2001) e o número de plantas e de espigas na colheita (Storck et al., 2002), têm sido utilizados buscando diminuir o erro experimental em ensaios de competição de cultivares de milho. Além disso, o uso de bordadura nas extremidades das fileiras (Cargnelutti Filho et al., 2003b) e a análise espacial (Cargnelutti Filho et al., 2003a) foram estudados visando reduzir o erro experimental em ensaios de competição de cultivares de milho. A desuniformidade relacionada ao número de plantas por parcela prejudica essas comparações e constitui um dos problemas básicos na análise e interpretação dos resultados experimentais (Schmildt et al., 2001; Cruz \& Carneiro, 2003). Por isto, tenta-se alcançar população uniforme pela semeadura em excesso, efetuando-se o desbaste para a população desejada quando as plantas estão em estádio inicial de crescimento. Entretanto, mesmo após esta prática, a parcela pode, algumas vezes, apresentar falhas causadas por fatores que fogem ao controle do pesquisador, tais como elevada precipitação pluvial, ação de pragas e doenças, com reflexos em análises estatísticas, em particular quando o caráter não é medido apenas nas plantas competitivas (Vencovsky \& Cruz, 1991). Assim, uma maneira de se considerar o efeito de população em análises estatísticas é incluí-lo no modelo como covariável.

Os métodos de correção da produtividade de parcelas com populações variadas têm sido aplicados com maior frequiência, por causa, principalmente, da disponibilidade de técnicas computacionais capazes de solucionar o volume de cálculos exigido. Trabalhos com dados simulados e de experimentos realizados no campo, envolvendo métodos de correção de estande para estimar produtividade, têm sido realizados (Morais et al., 1986; Vencovsky \& Cruz, 1991; Veronesi et al., 1995; Schmildt et al., 2001). De maneira geral, conforme estatísticas utilizadas na verificação da eficiência dos métodos, os resultados mostram-se promissores quanto à discriminação de cultivares, apontando uma tendência de melhorar a precisão experimental, principalmente nos métodos que utilizam o número de plantas como covariável.

Além da correção da produtividade de grãos, a análise de adaptabilidade e estabilidade constitui-se em uma das etapas finais de um programa de melhoramento de plantas, visando a indicação de cultivares para ambientes específicos de cultivo. A princípio, o método de correção dos dados da produtividade de grãos em experimentos inadequados quanto à população final de plantas, para ser considerado adequado, deve aumentar a discriminação de cultivares mas não afetar a indicação pela análise de adaptabilidade e estabilidade. Na aplicação de um método de correção, a interferência dessas correções nas estimativas dos parâmetros da análise de adaptabilidade e estabilidade usadas para as indicações de cultivares é pouco conhecida.

O objetivo deste trabalho foi verificar a interferência dos métodos de correção da produtividade de grãos de milho nos parâmetros de adaptabilidade e estabilidade de cultivares.

\section{Material e Métodos}

Foram usados os dados da produtividade de grãos de milho corrigidos a $13 \%$ de umidade $(\mathrm{Y})$ e da população final de plantas (X) de 31 ensaios de competição de cultivares de milho, realizados no Estado do Rio Grande do Sul, no ano agrícola 2002/2003. Esses ensaios classificaram-se em quatro grupos: dez ensaios de 36 cultivares no grupo dos estaduais de ciclo precoce, nove ensaios de 11 cultivares no grupo dos estaduais superprecoces, seis ensaios de 27 cultivares no grupo dos indicados precoces e seis ensaios de 18 cultivares 
no grupo dos indicados superprecoces. Na categoria dos ensaios estaduais, as cultivares avaliadas foram aquelas ainda não indicadas aos produtores, e as indicadas foram estudadas na categoria dos ensaios indicados. Em todos os ensaios, as cultivares foram delineadas em blocos casualizados, com três repetições, e as unidades experimentais constituídas de duas fileiras com 5,0 m de comprimento, espaçadas em $0,8 \mathrm{~m}$. Realizaram-se as semeaduras visando obter populações finais, respectivamente, de 55.000 e 65.000 plantas ha $^{-1}$ em relação aos ensaios dos ciclos precoce e superprecoce.

Em cada um dos 31 ensaios foram realizadas a análise da variância para as variáveis $\mathrm{Y}$ e X e a análise de covariância usando X como covariável. Nos casos em que o efeito da covariável $X$ foi significativo pelo teste de $\mathrm{F}(\alpha=0,05)$, a correção dos dados da produtividade de grãos de milho foi considerada adequada.

A correção ou não da produtividade de grãos em função da população de plantas foi realizada através de oito métodos, conforme Cruz \& Carneiro (2003), independentemente de ser adequado: sem correção (SC), correção por regra de três (RT), correção pelo método de Zuber (1942) (Z), correção pela covariância com a população média $(\mathrm{CM})$, correção pela covariância com a população ideal (CI), correção pelo método de Cruz (1971) (C), correção pelo método proposto por Vencovsky \& Cruz (1991) (VC) e correção estratificada, proposto por Schmildt et al. (2001) (CE).

Nos ensaios em que a análise de variância em relação à população final apresentou significância para o efeito de cultivar, o valor da estatística diferença mínima significativa entre as cultivares, pelo teste de Tukey a 5\% de probabilidade, foi utilizado para obtenção dos grupos de cultivares no método de correção estratificada.

A partir do coeficiente de variação experimental (CV) e dos valores da estatística $F$ calculada na análise da variância individual, estimaram-se as estatísticas $\mathrm{P}_{\mathrm{i}(100 /}$ CV) e $\mathrm{P}_{\mathrm{i}(\mathrm{F})}$, conforme Schmildt et al. (2001), e realizouse análise conjunta dos ambientes buscando identificar o método de correção que proporciona menores valores de coeficientes de variação e os maiores valores de $\mathrm{F}$.

A seguir, realizou-se a análise da variância conjunta da produtividade de grãos de milho sem e com a correção dos dados, separadamente para cada grupo de ensaios. Foram realizadas as análise de adaptabilidade e de estabilidade pelo método de Eberhart \& Russell (1966), usando os dados originais da produtividade de grãos de milho e os dados corrigidos em razão da população de plantas pelos métodos RT, Z, CM, CI, C, VC e
CE. Os parâmetros da análise de estabilidade usados foram a média $(\mu)$ e o coeficiente de regressão linear $(\beta)$, como medida de adaptabilidade e a variância dos desvios da regressão $\left(\sigma_{\delta}^{2}\right)$, como medida de estabilidade, para cada uma das cultivares.

A fim de verificar a magnitude da influência da correção pelos diferentes métodos, em relação aos dados sem correção, foram usadas as estatísticas desvio quadrático médio (DQM) para cada um dos parâmetros $\left(\mu, \beta\right.$ e $\left.\sigma_{\delta}^{2}\right)$ e índice de concordância (IC). Foram considerados os métodos $\mathrm{k}=1,2, \ldots, 7$ para as cultivares $\mathrm{i}=$ $1,2, \ldots, \mathrm{m}$, para cada um dos quatro grupos de ensaios, e as estimativas dos parâmetros $\hat{\mu}_{\mathrm{oi}}$ : estimativa da média da cultivar i usando os dados originais; $\hat{\mu}_{0}$ : estimativa da média geral usando os dados originais; $\hat{\mu}_{\mathrm{ki}}$ : estimativa da média da cultivar i usando os dados corrigidos pelo método $\mathrm{k} ; \hat{\mu}_{\mathrm{k}}$ : estimativa da média geral usando os dados corrigidos pelo método $\mathrm{k} ; \hat{\beta}_{\mathrm{oi}}$ : estimativa da adaptabilidade da cultivar i usando os dados originais; $\hat{\beta}_{\mathrm{ki}}$ : estimativa da adaptabilidade da cultivar i usando os dados corrigidos pelo método $\mathrm{k} ; \hat{\sigma}_{\delta \mathrm{oi}}^{2}$ : estimativa da estabilidade da cultivar i usando os dados originais e $\hat{\sigma}_{\text {cki }}^{2}$ : estimativa da estabilidade da cultivar i usando os dados corrigidos pelo método $\mathrm{k}$.

Foram estimados os seguintes índices de concordância: $\mathrm{IC}_{(\mu) \mathrm{k}}=\sum^{\mathrm{n}} \mathrm{C}_{(\mu) \mathrm{ki}} / \mathrm{n}$ : estimativa do índice de concordância do parâmetro $\mu$ para o método de correção k; $\mathrm{IC}_{(\beta) \mathrm{k}}=\sum_{\mathrm{i}=1}^{\mathrm{n}} \mathrm{C}_{(\beta) \mathrm{ki}} / \mathrm{n}$ : estimativa do índice de concordância do parấmetro $\beta$ para o método de correção k; $\mathrm{IC}_{\left(\sigma_{s}^{2}\right) \mathrm{k}}=\sum_{\mathrm{i}=1}^{\mathrm{n}} \mathrm{C}_{\left(\sigma_{s}^{2}\right) \mathrm{ki}} / \mathrm{n}$ : estimativa do índice de concordância do parâmetro $\sigma_{\delta}^{2}$ para o método de correção k; e $\mathrm{IC}_{\mathrm{k}}=\left\{\sum_{\mathrm{i}=1}^{\mathrm{n}}\left(\mathrm{C}_{(\mu) \mathrm{ki}}+\mathrm{C}_{(\beta) \mathrm{ki}}+\mathrm{C}_{\left(\sigma_{\delta}^{2}\right) \mathrm{ki}}\right)\right\} / 3 \mathrm{n}$ : índice de concordância médio para o método de correção $\mathrm{k}$, em que: $\mathrm{C}_{(\mu) \mathrm{ki}}=1$ se $\hat{\mu}_{\mathrm{oi}}>\hat{\mu}_{\mathrm{o}}$ e $\hat{\mu}_{\mathrm{ki}}>\hat{\mu}_{\mathrm{k}}$, se $\hat{\mu}_{\mathrm{oi}}<\hat{\mu}_{\mathrm{o}}$ e $\hat{\mu}_{\mathrm{ki}}<\hat{\mu}_{\mathrm{k}}$ ou se $\hat{\mu}_{\mathrm{oi}}=\hat{\mu}_{\mathrm{o}}$ e $\hat{\mu}_{\mathrm{ki}}=\hat{\mu}_{\mathrm{k}}$ e igual a zero em casos contrários; $\mathrm{C}_{(\beta) \mathrm{ki}}=1$ se $\hat{\beta}_{\mathrm{oi}}$ e $\hat{\beta}_{\mathrm{ki}}$ forem significativamente maiores do que um, se $\hat{\beta}_{\mathrm{oi}}$ e $\hat{\beta}_{\mathrm{ki}}$ forem significativamente menores do que um ou se $\hat{\beta}_{\mathrm{oi}}$ e $\hat{\beta}_{\mathrm{ki}}$ não forem significativamente diferentes de um e igual a zero em casos contrários, pelo teste $\mathrm{t}$ a $5 \%$ de probabilidade; $\mathrm{C}_{\left(\sigma_{\delta}^{2}\right) \mathrm{ki}}=1 \mathrm{se}$ $\hat{\sigma}_{\delta \mathrm{oi}}^{2}$ e $\hat{\sigma}_{\delta \mathrm{ki}}^{2}$ forem significativamente maiores do que zero, se $\hat{\sigma}_{\delta \mathrm{oi}}^{2}$ e $\hat{\sigma}_{\delta \mathrm{ki}}^{2}$ forem significativamente menores do que 
zero ou se $\hat{\sigma}_{\delta \mathrm{oi}}^{2} \mathrm{e} \hat{\sigma}_{\delta \mathrm{ki}}^{2}$ não forem significativamente diferentes de zero e igual a zero em casos contrários, pelo teste $\mathrm{F}$ a $5 \%$ de probabilidade e n: número de cultivares.

Foram estimados os seguintes desvios quadráticos médios: $\operatorname{DQM}_{(\mu) \mathrm{k}}=\sum_{\mathrm{i}=1}^{\mathrm{n}}\left(\hat{\mu}_{\mathrm{ki}}-\hat{\mu}_{\mathrm{oi}}\right)^{2} / \mathrm{n}$ : estimativa do desvio quadrático médio do parâmetro $\mu$ para o método de correção k; DQM $(\beta) \mathrm{k}=\sum_{\mathrm{i}=1}^{\mathrm{n}}\left(\hat{\beta}_{\mathrm{ki}}-\hat{\beta}_{\mathrm{oi}}\right)^{2} / \mathrm{n}$ : estimativa do desvio quadrático médio do parâmetro $\beta$ para o método de correção k; e DQM ${\left(\sigma_{\delta}^{2}\right) \mathrm{k}}_{\mathrm{i}=1}=\sum_{\delta \mathrm{\delta i}}^{\mathrm{n}}\left(\hat{\sigma}_{\delta \mathrm{oi}}^{2}\right)^{2} / \mathrm{n}$ : estimativa do desvio quadrático médio do parâmetro $\sigma_{\delta}^{2}$ para o método de correção $\mathrm{k}$.

As análises foram procedidas com o auxílio do programa GENES (Cruz, 2001) e do aplicativo Office Excel.

\section{Resultados e Discussão}

O efeito de cultivar em relação à produtividade de grãos foi significativo em $87 \%$ dos ensaios, passando para $94 \%$ com a análise de covariância (Tabela 1), o que evidencia uma técnica adequada para aumentar a discriminação de cultivares (Storck et al., 2000b, 2002; Cruz \& Carneiro, 2003).

Nos seis ensaios nos quais a estatística $\mathrm{F}(\alpha=0,05)$ foi significativa entre cultivares em relação à população de plantas, indicando diferenças no comportamento das cultivares submetidas a esses ambientes, a correção dos dados da produtividade de grãos deveria ser aplicada usando-se o método da correção estratificada (Schmildt et al., 2001). Porém, com a aplicação da análise de covariância nestes ensaios, o efeito da covariável também foi significativo, indicando que a produtividade de grãos deve ser ajustada. Nos demais 25 ensaios, as diferenças entre cultivares em relação à população de plantas não foram significativas, e, nestes casos, a correção da população de plantas é um procedimento adequado se houver significância da covariável (Steel et al., 1997; Ramalho et al., 2000; Cruz \& Carneiro, 2003).

No entanto, segundo Vencovsky \& Cruz (1991) e Veronesi et al. (1995), tem-se realizado a correção em todos os ambientes, utilizando-se a covariância para a população ideal, por permitir a estabilização dos valores ajustados ao redor desta população, que deve ser comum a todos os ensaios. Assim, considerando-se significante o uso da covariável população de plantas como critério para adequabilidade da correção dos da- dos da produtividade de grãos, constata-se $80 \%, 22 \%$, $66 \%$ e $100 \%$ dos ambientes com correção adequada, respectivamente, para ensaios dos grupos estaduais precoce, estaduais superprecoces, indicados precoces e indicados superprecoces (Tabela 1).

Os menores valores das estatísticas $\mathrm{P}_{\mathrm{i}(100 / \mathrm{CV})}$ e $\mathrm{P}_{\mathrm{i}(\mathrm{F})}$ indicam, respectivamente, menores valores do coeficiente de variação e maiores valores da estatística F calculada. Assim, para os 31 ensaios, os métodos basea-

Tabela 1. Efeito significativo $\left(\mathrm{H}_{1}\right)$ ou não significativo $\left(\mathrm{H}_{0}\right)$, a $5 \%$ de probabilidade de erro, para cultivares das variáveis produtividade de grãos, população de plantas, produtividade corrigida de grãos (PC) e da covariável população de plantas (Covar-Pop) e situação recomendada para os ensaios de diferentes grupos de milho.

\begin{tabular}{|c|c|c|c|c|c|}
\hline \multirow[t]{2}{*}{ Ensaios } & \multicolumn{2}{|c|}{ Análise de variância } & \multicolumn{2}{|c|}{ Análise de covariância } & \multirow[t]{2}{*}{ Situação $^{(1)}$} \\
\hline & Produtividade & População & $\mathrm{PC}$ & Covar-Pop & \\
\hline & \multicolumn{5}{|c|}{ Ensaios Estaduais Precoces } \\
\hline 1 & $\mathrm{H}_{1}$ & $\mathrm{H}_{1}$ & $\mathrm{H}_{1}$ & $\mathrm{H}_{1}$ & Sim \\
\hline 2 & $\mathrm{H}_{1}$ & $\mathrm{H}_{0}$ & $\mathrm{H}_{1}$ & $\mathrm{H}_{1}$ & Sim \\
\hline 3 & $\mathrm{H}_{1}$ & $\mathrm{H}_{1}$ & $\mathrm{H}_{1}$ & $\mathrm{H}_{1}$ & Sim \\
\hline 4 & $\mathrm{H}_{1}$ & $\mathrm{H}_{0}$ & $\mathrm{H}_{1}$ & $\mathrm{H}_{1}$ & Sim \\
\hline 5 & $\mathrm{H}_{1}$ & $\mathrm{H}_{0}$ & $\mathrm{H}_{1}$ & $\mathrm{H}_{1}$ & Sim \\
\hline 6 & $\mathrm{H}_{1}$ & $\mathrm{H}_{1}$ & $\mathrm{H}_{1}$ & $\mathrm{H}_{1}$ & Sim \\
\hline 7 & $\mathrm{H}_{1}$ & $\mathrm{H}_{0}$ & $\mathrm{H}_{1}$ & $\mathrm{H}_{0}$ & Não \\
\hline 8 & $\mathrm{H}_{1}$ & $\mathrm{H}_{0}$ & $\mathrm{H}_{1}$ & $\mathrm{H}_{1}$ & Sim \\
\hline 9 & $\mathrm{H}_{1}$ & $\mathrm{H}_{0}$ & $\mathrm{H}_{1}$ & $\mathrm{H}_{0}$ & Não \\
\hline 10 & $\mathrm{H}_{1}$ & $\mathrm{H}_{0}$ & $\mathrm{H}_{1}$ & $\mathrm{H}_{1}$ & Sim \\
\hline \multicolumn{6}{|c|}{ Ensaios Estaduais Superprecoces } \\
\hline 1 & $\mathrm{H}_{1}$ & $\mathrm{H}_{0}$ & $\mathrm{H}_{1}$ & $\mathrm{H}_{0}$ & Não \\
\hline 2 & $\mathrm{H}_{1}$ & $\mathrm{H}_{0}$ & $\mathrm{H}_{1}$ & $\mathrm{H}_{0}$ & Não \\
\hline 3 & $\mathrm{H}_{1}$ & $\mathrm{H}_{0}$ & $\mathrm{H}_{1}$ & $\mathrm{H}_{0}$ & Não \\
\hline 4 & $\mathrm{H}_{1}$ & $\mathrm{H}_{0}$ & $\mathrm{H}_{1}$ & $\mathrm{H}_{0}$ & Não \\
\hline 5 & $\mathrm{H}_{1}$ & $\mathrm{H}_{1}$ & $\mathrm{H}_{1}$ & $\mathrm{H}_{1}$ & Sim \\
\hline 6 & $\mathrm{H}_{0}$ & $\mathrm{H}_{1}$ & $\mathrm{H}_{1}$ & $\mathrm{H}_{1}$ & Sim \\
\hline 7 & $\mathrm{H}_{1}$ & $\mathrm{H}_{0}$ & $\mathrm{H}_{1}$ & $\mathrm{H}_{0}$ & Não \\
\hline 8 & $\mathrm{H}_{1}$ & $\mathrm{H}_{0}$ & $\mathrm{H}_{1}$ & $\mathrm{H}_{0}$ & Não \\
\hline 9 & $\mathrm{H}_{1}$ & $\mathrm{H}_{0}$ & $\mathrm{H}_{1}$ & $\mathrm{H}_{0}$ & Não \\
\hline \multicolumn{6}{|c|}{ Ensaios Indicados Precoces } \\
\hline 1 & $\mathrm{H}_{1}$ & $\mathrm{H}_{0}$ & $\mathrm{H}_{1}$ & $\mathrm{H}_{1}$ & Sim \\
\hline 2 & $\mathrm{H}_{1}$ & $\mathrm{H}_{0}$ & $\mathrm{H}_{1}$ & $\mathrm{H}_{1}$ & Sim \\
\hline 3 & $\mathrm{H}_{1}$ & $\mathrm{H}_{0}$ & $\mathrm{H}_{1}$ & $\mathrm{H}_{1}$ & Sim \\
\hline 4 & $\mathrm{H}_{1}$ & $\mathrm{H}_{0}$ & $\mathrm{H}_{1}$ & $\mathrm{H}_{0}$ & Não \\
\hline 5 & $\mathrm{H}_{1}$ & $\mathrm{H}_{0}$ & $\mathrm{H}_{1}$ & $\mathrm{H}_{0}$ & Não \\
\hline 6 & $\mathrm{H}_{1}$ & $\mathrm{H}_{0}$ & $\mathrm{H}_{1}$ & $\mathrm{H}_{1}$ & Sim \\
\hline \multicolumn{6}{|c|}{ Ensaios Indicados Superprecoces } \\
\hline 1 & $\mathrm{H}_{1}$ & $\mathrm{H}_{0}$ & $\mathrm{H}_{1}$ & $\mathrm{H}_{1}$ & Sim \\
\hline 2 & $\mathrm{H}_{0}$ & $\mathrm{H}_{0}$ & $\mathrm{H}_{1}$ & $\mathrm{H}_{1}$ & Sim \\
\hline 3 & $\mathrm{H}_{1}$ & $\mathrm{H}_{1}$ & $\mathrm{H}_{1}$ & $\mathrm{H}_{1}$ & Sim \\
\hline 4 & $\mathrm{H}_{0}$ & $\mathrm{H}_{0}$ & $\mathrm{H}_{0}$ & $\mathrm{H}_{1}$ & Sim \\
\hline 5 & $\mathrm{H}_{0}$ & $\mathrm{H}_{0}$ & $\mathrm{H}_{0}$ & $\mathrm{H}_{1}$ & Sim \\
\hline 6 & $\mathrm{H}_{1}$ & $\mathrm{H}_{0}$ & $\mathrm{H}_{1}$ & $\mathrm{H}_{1}$ & Sim \\
\hline
\end{tabular}

${ }^{(1)}$ Sim: fazer a correção da produtividade de grãos quando o efeito da covariável população de plantas for significativo $\left(\mathrm{H}_{1}\right)$; não: não fazer a correção da produtividade de grãos quando o efeito da covariável população de plantas for não significativo $\left(\mathrm{H}_{0}\right)$. 
dos em análise de covariância (CM, CI, VC e CE) mostraram-se eficientes em melhorar a discriminação de cultivares de milho, e os métodos RT, Z e C, mostraram-se pouco adequados à correção dos dados (Tabela 2), concordando com Schmildt et al. (2001). Os métodos CM (Morais et al., 1986), CI (Morais et al., 1986; Vencovsky \& Cruz, 1991; Veronesi et al., 1995) e VC (Vencovsky \& Cruz, 1991; Veronesi et al., 1995) proporcionaram melhores resultados em termos de correção da produtividade de grãos.

A análise da variância conjunta mostrou haver interação cultivares $x$ ambiente significativa nos 32 casos (4 grupos de ensaios x dados sem e com sete métodos de correção), mostrando que a indicação de cultivares por meio da análise de adaptabilidade e estabilidade é um procedimento adequado.

No grupo de ensaios estaduais superprecoces, conforme critério adotado, recomenda-se não realizar correção dos dados da produtividade de grãos em 7 de 9 ensaios (78\%), podendo explicar os menores índices de concordância dos parâmetros de adaptabilidade e estabilidade dos diferentes métodos de correção em relação aos dados originais (média dos sete métodos $=0,88$ ). Portanto, quando a correção não é adequada, poderá acarretar maiores alterações na indicação de cultivares, pela análise de adaptabilidade e estabilidade (Tabela 3). No grupo de ensaios indicados superprecoces, com recomendação de correção em todos os ambientes, são observados menores índices de discordância em relação aos dados sem correção (média dos sete métodos = $5 \%$ ). Portanto, pode-se inferir que nos casos em que a correção é adequada, além do aumento da discriminação das cultivares, os métodos de correção acarretam menores alterações na indicação de cultivares por meio dos parâmetros de adaptabilidade e estabilidade $\left(\mu, \beta \mathrm{e} \sigma_{\delta}^{2}\right)$.
O índice de concordância médio, envolvendo os três parâmetros da análise de estabilidade $\left(\mu, \beta\right.$ e $\left.\sigma_{\delta}^{2}\right)$ calculado pelo método de correção da covariância com a população média (CM) igual a $94 \%$ na média dos quatro grupos de ensaios (Tabela 3), foi o maior entre os sete métodos, e apresentou os menores valores de DQM dos parâmetros $\left(\mu, \beta\right.$ e $\left.\sigma_{\delta}^{2}\right)$, significando que as conclusões após a correção prévia dos dados são as mais próximas das alcançadas com os dados originais. Os menores valores dos DQM já eram esperados no método $\mathrm{CM}$, já que ele estima os valores próximos à média das cultivares, sendo, portanto, o que menos altera a magnitude das estimativas. Porém, principalmente quando se dispõe de um conjunto de ensaios com a mesma população de plantas, a correção feita no estande ideal (CI) tem sido preferida, pois eles apresentam, geralmente, um particular estande médio, e a correção feita assim conduz a valores da produtividade de grãos para um referencial de estande variado. Desse modo, ao realizar a análise conjunta (adaptabilidade e estabilidade) dos ensaios, é gerada uma diferença ambiental adicional, devido à correção diferenciada pelo método $\mathrm{CM}$ (Cruz \& Carneiro, 2003). Em outro extremo, o menor índice de concordância $(0,88)$ e os maiores desvios quadráticos médios de $\mu, \beta$ e $\sigma_{\delta}^{2}$ revelam que o método de correção por regra de três (RT) é o menos adequado, ocasionando maiores alterações na indicação de cultivares. Além disso, os valores altos das estatísticas $\mathrm{P}_{\mathrm{i}(100 / \mathrm{CV})}$ e $\mathrm{P}_{\mathrm{i}(\mathrm{F})}$ (Tabela 2) reforçam que este método de correção não é adequado para melhorar a discriminação de cultivares, concordando com Vencovsky \& Cruz (1991) e Veronesi et al. (1995), que constataram a não existência de proporcionalidade linear entre o número de plantas e a produtividade de grãos em estudos comparativos de milho, podendo a produtividade de di-

Tabela 2. Valores das estatísticas $\mathrm{P}_{\mathrm{i}(100 / \mathrm{CV})}$ e $\mathrm{P}_{\mathrm{i}(\mathrm{F})}$ em relação à análise da produtividade de grãos sem e com correção da produtividade para população de plantas, usando diversos métodos, em 31 ensaios de competição de cultivares de milho.

\begin{tabular}{llllllllc}
\hline Estatísticas & \multicolumn{7}{c}{ Métodos de correção $^{(1)}$} \\
\cline { 2 - 9 } & \multicolumn{1}{c}{$\mathrm{SC}$} & $\mathrm{RT}$ & $\mathrm{Z}$ & $\mathrm{CM}$ & $\mathrm{CI}$ & $\mathrm{C}$ & \multicolumn{1}{c}{$\mathrm{VC}$} & $\mathrm{CE}$ \\
\hline $\mathrm{P}_{\mathrm{i}(100 / \mathrm{CV})}$ & 1,7923 & 1,7312 & 0,7357 & 0,3002 & 0,1521 & 0,8259 & 0,3848 & 0,2445 \\
$\mathrm{P}_{\mathrm{i}(\mathrm{F})}$ & 2,43 & 9,81 & 4,11 & 1,20 & 1,20 & 1,30 & 1,15 & 0,44 \\
\hline
\end{tabular}

(1)SC: sem correção; RT: correção por regra de três; Z: correção pelo método de Zuber (1942); CM: correção pela covariância com população média; CI: correção pela covariância com população ideal; C: correção pelo método de Cruz (1971); VC: correção pelo método proposto por Vencovsky \& Cruz (1991); CE: correção estratificada. 
ferentes cultivares ser superestimada por este método. Os demais métodos de correção situam-se numa faixa intermediária (Tabela 3).

As estatísticas desvio quadrático médio (DQM) e índice de concordância (IC) revelam, de modo geral, que os métodos de correção acarretaram alterações nos três parâmetros $\left(\mu, \beta\right.$ e $\left.\sigma_{\hat{o}}^{2}\right)$, sendo verificada maior influência na medida de estabilidade $\left(\sigma_{\delta}^{2}\right)$ (menores IC e maiores DQM) e menor influência na média $(\mu)$ e na adaptabilidade $(\beta)$ (maiores IC e DQM menores). Portanto,

Tabela 3. Estimativas do índice de concordância dos parâmetros $\mu, \beta$ e $\sigma_{\delta}^{2}$, do índice de concordância médio e desvio quadrático médio dos parâmetros $\mu, \beta$ e $\sigma_{\delta}^{2}$, para o método de correção $\mathrm{k}$, em quatro grupos de ensaios de cultivares de milho e na média dos grupos.

\begin{tabular}{|c|c|c|c|c|c|c|c|}
\hline \multirow[t]{2}{*}{ Método $^{(1)}(\mathrm{k})$} & \multicolumn{4}{|c|}{ Índice de concordância } & \multicolumn{3}{|c|}{ Desvio quadrático médio } \\
\hline & $\mu$ & $\beta$ & $\sigma_{\delta}^{2}$ & Médio & $\mu$ & $\beta$ & $\sigma_{\delta}^{2}$ \\
\hline & \multicolumn{7}{|c|}{ Ensaios Estaduais Precoces } \\
\hline RT & 1,00 & 0,75 & 0,81 & 0,85 & 0,0924 & 0,0089 & 0,1584 \\
\hline $\mathrm{Z}$ & 1,00 & 0,89 & 0,83 & 0,91 & 0,0453 & 0,0041 & 0,0711 \\
\hline $\mathrm{CM}$ & 1,00 & 0,89 & 0,86 & 0,92 & 0,0152 & 0,0016 & 0,0530 \\
\hline $\mathrm{CI}$ & 1,00 & 0,89 & 0,89 & 0,93 & 0,0653 & 0,0022 & 0,0877 \\
\hline $\mathrm{C}$ & 1,00 & 0,86 & 0,89 & 0,92 & 0,0581 & 0,0028 & 0,0771 \\
\hline $\mathrm{VC}$ & 1,00 & 0,86 & 0,89 & 0,92 & 0,0694 & 0,0022 & 0,0850 \\
\hline \multirow[t]{2}{*}{$\mathrm{CE}$} & 1,00 & 0,83 & 0,86 & 0,90 & 0,0656 & 0,0023 & 0,0875 \\
\hline & \multicolumn{7}{|c|}{ Ensaios Estaduais Superprecoces } \\
\hline RT & 1,00 & 0,82 & 0,73 & 0,85 & 0,1944 & 0,0020 & 0,0194 \\
\hline Z & 1,00 & 0,82 & 0,82 & 0,88 & 0,0953 & 0,0009 & 0,0128 \\
\hline $\mathrm{OM}$ & 1,00 & 1,00 & 0,82 & 0,94 & 0,0188 & 0,0003 & 0,0159 \\
\hline CI & 1,00 & 0,82 & 0,82 & 0,88 & 0,0575 & 0,0005 & 0,0186 \\
\hline $\mathrm{C}$ & 1,00 & 0,73 & 0,91 & 0,88 & 0,0438 & 0,0007 & 0,0436 \\
\hline $\mathrm{VC}$ & 1,00 & 0,82 & 0,82 & 0,88 & 0,0545 & 0,0006 & 0,0141 \\
\hline \multirow[t]{2}{*}{$\mathrm{CE}$} & 1,00 & 0,91 & 0,73 & 0,88 & 0,0280 & 0,0005 & 0,0356 \\
\hline & \multicolumn{7}{|c|}{ Ensaios Indicados Precoces } \\
\hline RT & 1,00 & 0,93 & 0,74 & 0,89 & 0,0541 & 0,0345 & 1,4396 \\
\hline $\mathrm{Z}$ & 1,00 & 0,93 & 0,78 & 0,90 & 0,0265 & 0,0116 & 0,4810 \\
\hline $\mathrm{CM}$ & 1,00 & 0,93 & 0,89 & 0,94 & 0,0154 & 0,0047 & 0,0384 \\
\hline CI & 1,00 & 0,96 & 0,85 & 0,94 & 0,0169 & 0,0065 & 0,0410 \\
\hline $\mathrm{C}$ & 1,00 & 0,93 & 0,81 & 0,91 & 0,0222 & 0,0157 & 0,1215 \\
\hline $\mathrm{VC}$ & 1,00 & 0,93 & 0,85 & 0,93 & 0,0148 & 0,0075 & 0,0676 \\
\hline \multirow[t]{2}{*}{$\mathrm{CE}$} & 1,00 & 0,96 & 0,85 & 0,94 & 0,0169 & 0,0065 & 0,0410 \\
\hline & \multicolumn{7}{|c|}{ Ensaios Indicados Superprecoces } \\
\hline $\mathrm{RT}$ & 0,94 & 0,89 & 1,00 & 0,94 & 0,2753 & 0,0058 & 0,0448 \\
\hline $\mathrm{Z}$ & 0,94 & 0,94 & 1,00 & 0,96 & 0,1349 & 0,0024 & 0,0239 \\
\hline $\mathrm{CM}$ & 1,00 & 0,94 & 0,94 & 0,96 & 0,0332 & 0,0011 & 0,0614 \\
\hline $\mathrm{CI}$ & 1,00 & 0,94 & 0,94 & 0,96 & 0,1779 & 0,0019 & 0,0674 \\
\hline $\mathrm{C}$ & 0,94 & 0,83 & 1,00 & 0,93 & 0,1648 & 0,0054 & 0,0403 \\
\hline $\mathrm{VC}$ & 0,94 & 0,94 & 1,00 & 0,96 & 0,1636 & 0,0039 & 0,0372 \\
\hline \multirow[t]{2}{*}{$\mathrm{CE}$} & 1,00 & 0,94 & 0,94 & 0,96 & 0,1671 & 0,0020 & 0,0771 \\
\hline & \multicolumn{7}{|c|}{ Médias dos quatro grupos de ensaios } \\
\hline RT & 0,98 & 0,85 & 0,82 & 0,88 & 0,1540 & 0,0128 & 0,4155 \\
\hline $\mathrm{Z}$ & 0,98 & 0,89 & 0,86 & 0,91 & 0,0755 & 0,0047 & 0,1472 \\
\hline $\mathrm{CM}$ & 1,00 & 0,94 & 0,88 & 0,94 & 0,0206 & 0,0019 & 0,0421 \\
\hline CI & 1,00 & 0,90 & 0,87 & 0,93 & 0,0794 & 0,0027 & 0,0536 \\
\hline $\mathrm{C}$ & 0,98 & 0,84 & 0,90 & 0,91 & 0,0722 & 0,0062 & 0,0706 \\
\hline $\mathrm{VC}$ & 0,98 & 0,88 & 0,89 & 0,92 & 0,0755 & 0,0035 & 0,0509 \\
\hline $\mathrm{CE}$ & 1,00 & 0,91 & 0,84 & 0,92 & 0,0694 & 0,0028 & 0,0603 \\
\hline
\end{tabular}

${ }^{(1)}$ RT: correção por regra de três; Z: correção pelo método de Zuber (1942); CM: correção pela covariância com população média; CI: correção pela covariância com população ideal; C: correção pelo método de Cruz (1971); VC: correção pelo método proposto por Vencovsky \& Cruz (1991); CE: correção estratificada. 
pode-se inferir que qualquer método de correção da produtividade de grãos leva a conclusões diferentes das alcançadas com os dados originais (Tabela 3).

Considerando que os métodos que envolvem análise de covariância para a população ideal (CI), covariância para a população média $(\mathrm{CM})$ e outros que se baseiam em um fator de compensação estimado a partir dos dados experimentais (VC), além da correção estratificada (CE), proporcionam maiores ganhos nas discriminações de cultivares e acarretam menores discordâncias e desvios quadráticos médios dos parâmetros $\left(\mu, \beta\right.$ e $\left.\sigma_{\delta}^{2}\right)$, estes devem ser preferidos quando a correção prévia dos dados da produtividade de grãos em função da população de plantas for realizada. As discordâncias médias dos quatro grupos de ensaios nesses quatro métodos de correção foi de $6 \%, 7 \%, 8 \%$ e $8 \%$, respectivamente, para os métodos $\mathrm{CM}, \mathrm{CI}, \mathrm{VC}$ e $\mathrm{CE}$, indicando poucas alterações nos parâmetros $\mu, \beta$ e $\sigma_{\delta}^{2}$, podendo ser considerado como critério para escolha do método de correção aquele que proporciona maior discriminação das cultivares.

\section{Conclusões}

1. Os sete métodos de correção da produtividade de grãos proporcionam diferentes níveis de discordância nos parâmetros de adaptabilidade e estabilidade de cultivares de milho.

2. Os métodos de correção por análise de covariância para a população média, correção por análise de covariância para a população ideal, correção pelo método proposto por Vencovsky \& Cruz e correção estratificada são os que acarretam menores discordâncias dos parâmetros de adaptabilidade e estabilidade de cultivares.

\section{Agradecimentos}

Ao CNPq, pela concessão de bolsa a Lindolfo Storck; ao Engenheiro Agrônomo José Paulo Guadagnin e demais pesquisadores que auxiliaram na realização dos experimentos, pela cessão dos dados.

\section{Referências}

CARGNELUTTI FILHO, A.; STORCK, L.; LUCIO, A.D. Ajustes de quadrado médio do erro em ensaios de competição de cultivares de milho pelo método de Papadakis. Pesquisa Agropecuária Brasileira, v.38, p.467-473, 2003a.
CARGNELUTTI FILHO, A.; STORCK, L.; LUCIO, A.D.; CARVALHO, M.P. de; SANTOS, P.M dos. A precisão experimental relacionada ao uso de bordaduras nas extremidades das fileiras em ensaios de milho. Ciência Rural, v.33, p.607-614, 2003b.

CONAGIN, A.; IGUE, T.; NAGAI, V. Tabelas para determinação do número de repetições no planejamento de experimentos. Campinas: Instituto Agronômico, 1995. 17p. (Boletim Científico, 34).

CRUZ, C.D. Programa GENES - versão Windows: aplicativo computacional em genética e estatística. Viçosa: UFV, 2001. 648p.

CRUZ, C.D.; CARNEIRO, P.C.S. Modelos biométricos aplicados ao melhoramento genético. Viçosa, MG: UFV, 2003. 579p.

CRUZ, V.F. da. Estudo sobre a correção de produção de parcelas em ensaios com milho. 1971. 143p. Tese(Doutorado) - Escola Superior de Agricultura Luiz de Queiroz, Piracicaba.

EBERHART, S.A.; RUSSELL, W.A. Stability parameters for comparing varieties. Crop Science, v.6, p.36-40, 1966.

FEIJÓ, S.; STORCK, L.; LÚCIO, A.D.; LOVATO, C.; MARTIN, T.N. Melhoria da qualidade de ensaios de avaliação de cultivares de milho através de indicadores de fertilidade do solo. Ciência Rural, v.31, p.225-230, 2001.

GOMES, F.P. A importância do número de repetições nos experimentos. Revista de Agricultura, v.69, p.243-245, 1994.

LOPES, S.J. Avaliação do efeito de diferentes formas de adubação sobre a precisão de ensaios de milho. 1993. 72p. Dissertação (Mestrado) - Universidade Federal de Santa Maria, Santa Maria.

LOPES, S.J.; STORCK, L. A precisão experimental para diferentes manejos na cultura do milho. Ciência Rural, v.25, p.49-53, 1995.

LÚCIO, A.D. Parâmetros da precisão experimental das principais culturas anuais do Estado do Rio Grande do Sul. 1997. 64p. Dissertação (Mestrado) - Universidade Federal de Santa Maria, Santa Maria.

MARQUES, D.G. As pressuposições e a precisão dos ensaios de competição de cultivares de milho no Estado do Rio Grande do Sul. 1999. 42p. Dissertação (Mestrado) - Universidade Federal de Santa Maria, Santa Maria.

MORAIS, A.R.; OLIVEIRA, A.C.; CRUZ, J.C. Comparação de métodos de correção de produções de milho em parcelas experimentais. Relatório Técnico Anual do CNPMS: 1980-1984, p.130-132, 1986.

RAMALHO, M.A.P.; FERREIRA, D.F.; OLIVEIRA, A.C. Experimentação em genética e melhoramento de plantas. Lavras: Ufla, 2000. 326p.

SCAPIM, C.A.; CARVALHO, C.G.P. de; CRUZ, C.D. Uma proposta de classificação dos coeficientes de variação para a cultura do milho. Pesquisa Agropecuária Brasileira, v.30, p.683-686, 1995.

SCHMILDT, E.R.; CRUZ, C.D.; ZANUNCIO, J.C.; PEREIRA, P.R.G.; FERRÃO, R.G. Avaliação de métodos de correção de estande para estimar a produtividade em milho. Pesquisa Agropecuária Brasileira, v.36, p.1011-1018, 2001.

STEEL, R.G.D.; TORRIE, J.H.; DICKEY, D.A. Principles and 
procedures of statistics. $3^{\text {rd }}$ ed. New York: McGraw Hill Book, 1997. 666p.

STORCK, L.; GARCIA, D.C.; LOPES, S.J.; ESTEFANEL, V. Experimentação vegetal. Santa Maria: UFSM, 2000a. 198p.

STORCK, L.; LOPES, S.J.; MARQUES, D.G.; TISOTT, C.A.; ROS, C.A. da. Análise de covariância para melhoria da capacidade de discriminação em ensaios de cultivares de milho. Pesquisa Agropecuária Brasileira, v.35, p.1311-1316, $2000 \mathrm{~b}$.

STORCK, L.; LÚCIO, A.D.; MARTIN, T.N.; LORENZ, L.H.; SOUZA, M.F. de. Diferenças de produtividade de grãos entre cultivares indicados de milho, ajustado para um mesmo número de plantas ou de espigas. Ciência Rural, v.32, p.745-750, 2002.

VENCOVSKY, R.; CRUZ, C.D. Comparação de métodos de correção do rendimento de parcelas com estandes variados: I. Dados simulados. Pesquisa Agropecuária Brasileira, v.26, p.647-657, 1991.

VERONESI, J.A.; CRUZ, C.D.; CORRÊA, L.A.; SCAPIM, C.A. Comparação de métodos de ajuste do rendimento de parcelas com estandes variados. Pesquisa Agropecuária Brasileira, v.30, p.169174, 1995.

ZUBER, M.S. Relative efficiency of incomplete block designs using corn uniform trial data. Journal of the American Society of Agronomy, v.34, p.30-47, 1942.

Recebido em 4 de março de 2004 e aprovado em 2 de julho de 2004 